\title{
DEMOKRASI YANG BERETIKA DAN BERBUDAYA PANCASILA PADA PILKADA SERENTAK TAHUN 2018
}

\author{
'Shohibul Anshor Siregar \\ Dosen FISIP UMSU. Koordinator Umum Pengembangan Basis Sosial \\ Inisiatif \& Swadaya ("nBASIS)
}

'Dialog Demokrasi

Pemerintah Provinsi Sumatera Utara BADAN KESATUAN BANGSA DAN POLITIK Medan, 28 November 2017 

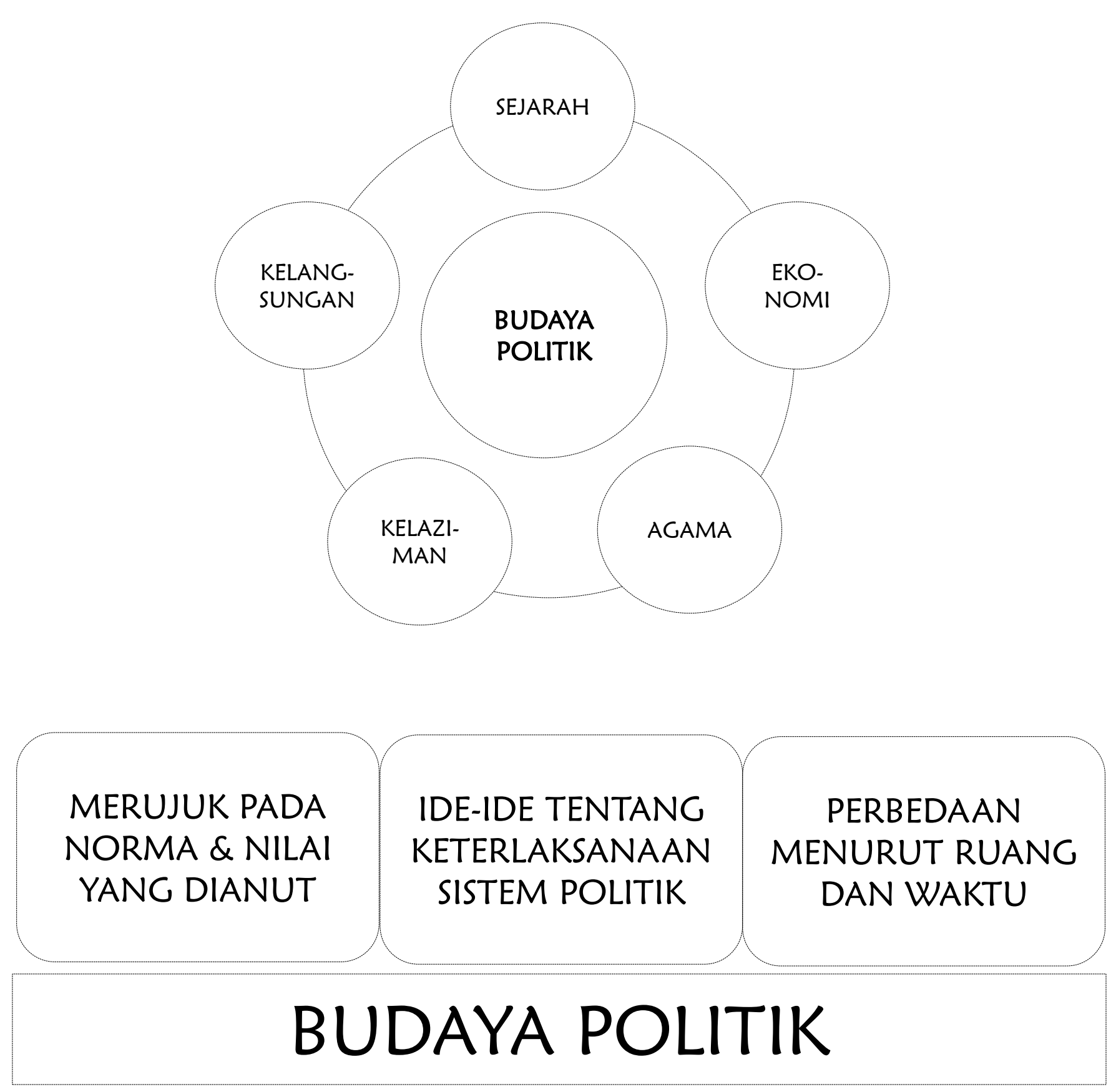


\section{POLA PERILAKU SUATU MASYARAKAT}

- DALAM KEHIDUPAN BERNEGARA

- PENYELENGGARAAN ADMINISTRASI NEGARA, POLITIK BUDAYA PEMERINTAHAN, POLITIK DAN HUKUM

- NORMA KEBIASAAN YANG DIHAYATI OLEH SELURUH ANGGOTA MASYARAKAT

DALAM MEMANDANG KEKUASAAN
SUATU SISTEM NILAI BERSAMA

KESADARAN

UNTUK

BERPARTISIPASI

DALAM

PENGAMBILAN

KEPUTUSAN

KOLEKTIF

- TANGGUNG

JAWAB DALAM

PENENTUAN

KEBIJAKAN PUBLIK

UNTUK

MASYARAKAT

SELURUHNYA
VARIAN (BENTUK)

- APATIS (TIDAK ACUH, MASA BODOH, DAN PASIF);

- MOBILISASI (DIDORONG ATAU SENGAJA DIMOBILISASI)

- PARTISIPATIF (AKTIF) 


\section{MASALAH DASAR DALAM HIDUP \& ORIENTASI NILAI BUDAYA}

\begin{tabular}{|l|l|l|l|}
\hline \multicolumn{1}{|c|}{$\begin{array}{c}\text { MASALAH DASAR } \\
\text { DALAM IDUP }\end{array}$} & \multicolumn{3}{|c|}{ ORIENTASI NILAI BUDAYA } \\
\cline { 2 - 4 } HAKEKAT HIDUP & $\begin{array}{l}\text { KIDUP INI TIDAK } \\
\text { BURUK }\end{array}$ & HIDUP INI BAIK & $\begin{array}{l}\text { TRANSISI } \\
\text { HARUSI SUKAR HIDUP } \\
\text { DIPERJUANGKAN }\end{array}$ \\
\hline HAKEKATKERJA & $\begin{array}{l}\text { KELANGSUNGAN } \\
\text { HIDUP }\end{array}$ & $\begin{array}{l}\text { KEDUDUKAN DAN } \\
\text { KEHORMATAN }\end{array}$ & $\begin{array}{l}\text { MEMPERTINGGI } \\
\text { PRESTISE }\end{array}$ \\
\hline $\begin{array}{l}\text { HUBUNGAN MANUSIA } \\
\text { DENGAN WAKTU }\end{array}$ & $\begin{array}{l}\text { ORIENTASI MASA } \\
\text { LALU }\end{array}$ & $\begin{array}{l}\text { ORIENTASI MASA } \\
\text { KINI }\end{array}$ & $\begin{array}{l}\text { ORIENTASI KE MASA } \\
\text { DEPAN }\end{array}$ \\
\hline $\begin{array}{l}\text { HUBUNGAN MANUSIA } \\
\text { DENGAN ALAM }\end{array}$ & $\begin{array}{l}\text { TUNDUK KEPADA } \\
\text { ALAM }\end{array}$ & $\begin{array}{l}\text { SELARAS DENGAN } \\
\text { ALAM }\end{array}$ & $\begin{array}{l}\text { MENGUASAI } \\
\text { ALAM }\end{array}$ \\
\hline $\begin{array}{l}\text { HUBUNGAN MANUSIA } \\
\text { DENGAN SESAMA }\end{array}$ & VERTIKAL & HORISONTAL & INDIVIDUAL \\
\hline
\end{tabular}




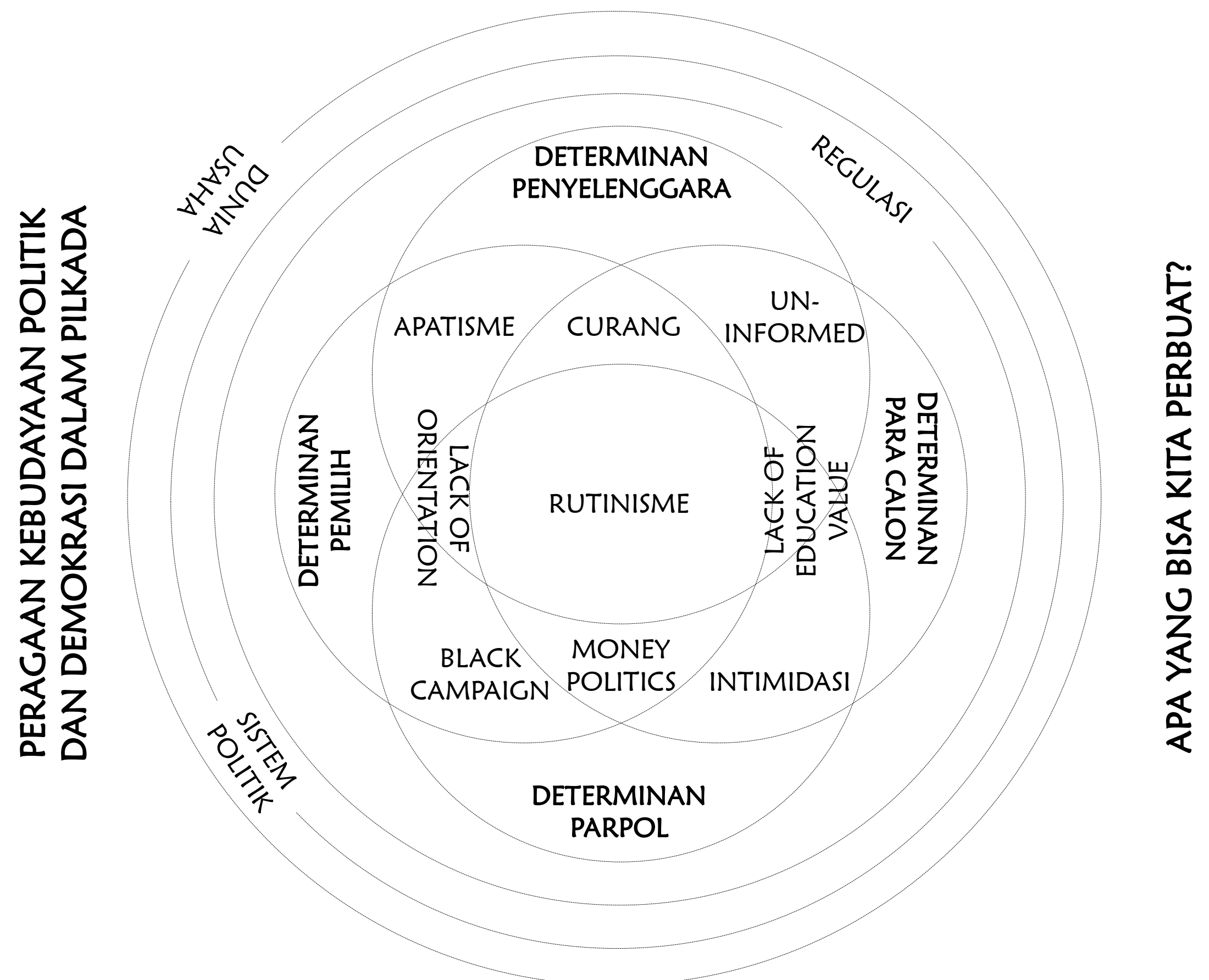




\section{TEORI STRAIN ROBERT KING MERTON}

CARA-CARA YANG SUDAH DILEMBAGAKAN

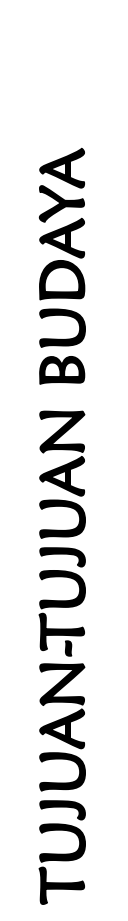

TERTOLAK

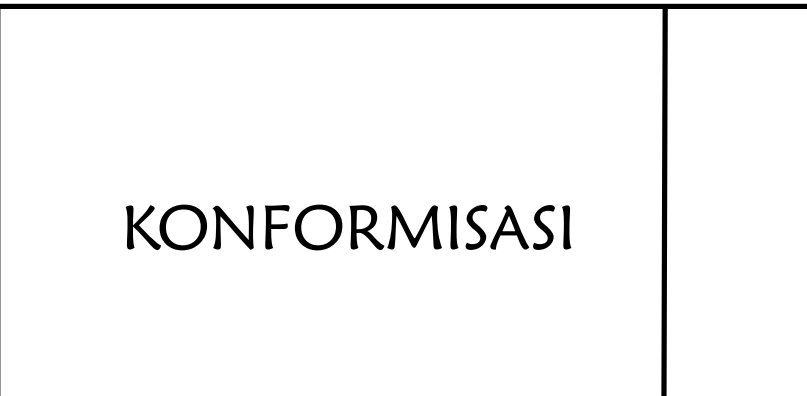

INOVASI
TERTERIMA

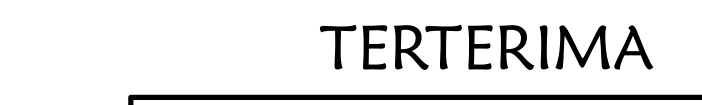

KONFORMISASI

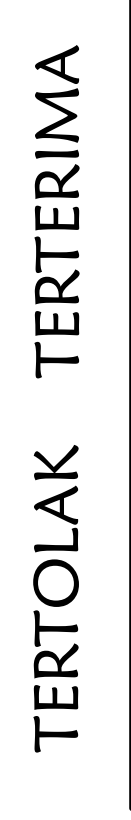

BILA INDIVIDU DIHADAPKAN PADA KESENJANGAN ANTARA 'APA YANG SEHARUSNYA DAN 'REALITAS SOSIAL APA ADANYA', ORANG ITU AKAN MERASA TEGANG DAN MEMILIKI PILIHAN ANTARA LIMA MODE ADAPTASI.
BAHWA

MASYARAKAT

TRANSISI SEPERTI INDONESIA TIDAK SERTA-MERTA MAMPU MENYEDIAKAN SARANA UNTUK MENCAPAI TUJUAN BUDAYA (ADIL DAN MAKMUR).

CARA BARU 
PERTAMA, KONFORMISASI. ORANG YANG PERCAYA PADA CARA NORMATIF UNTUK MENCAPAI TUJUAN SECARA SAH DAN MENGIKUTI ATURAN MASYARAKAT.

KEDUA, RITUALISME. INDIVIDU YANG BERHENTI BERUSAHA MENCAPAI TUJUAN NAMUN PERCAYA DENGAN MENGGUNAKAN CARA YANG SAH UNTUK MENCAPAI TUJUAN. (IKUTI PERATURAN). MISALNYA, TETAP DALAM USAHA DAN PEKERJAAN YANG SEBETULNYA SUDAH MERUPAKAN JALAN BUNTU.

KETIGA, INNORVATORS. ORANG YANG MENERIMA TUJUAN BUDAYA MASYARAKAT NAMUN MENOLAK METODE KONVENSIONAL, DAN MEMILIH MENGGUNAKAN CARA YANG BERBEDA (KRIMINAL).

KEEMPAT, RETRETIS. INDIVIDU YANG MENOLAK TUJUAN BUDAYA DAN CARA YANG DITERIMA UNTUK MENCAPAI TUJUAN. MEREKA MENGHINDARI TUJUAN DAN SARANA YANG DITETAPKAN OLEH MASYARAKAT DENGAN KEKUATAN KONTRA-BUDAYA MEREKA SENDIRI.

KELIMA, MEMBERONTAK. PEMBERONTAK TIDAK HANYA MENOLAK TUJUAN BUDAYA YANG TELAH MAPAN DAN CARA YANG DITERIMA UNTUK MENCAPAI TUJUAN, TETAPI MEREKA MENGGANTIKAN TUJUAN BARU DAN CARA BARU UNTUK MENCAPAINYA UNTUK MENGHASILKAN PERUBAHAN REVOLUSIONER DAN MENCIPTAKAN MASYARAKAT BARU. 


\section{TEORI STRAIN ROBERT KING MERTON}

\begin{tabular}{|l|c|c|}
\hline PILIHAN ADAPTASI & $\begin{array}{c}\text { TUJUAN } \\
\text { BUDAYA }\end{array}$ & $\begin{array}{c}\text { CARA-CARA } \\
\text { TERLEMBAGA }\end{array}$ \\
\hline KONFORMASI & + & + \\
\hline INOVASI & + & - \\
\hline RITUALISME & - & + \\
\hline RETRETIS & - & - \\
\hline BERONTAK & \pm & \pm \\
\hline
\end{tabular}




\section{PENUTUP}

- DEMOKRASI YANG BERETIKA DAN BERBUDAYA PANCASILA ADALAH SEBUAH CITA-CITA IDEAL YANG PERWUJUDANNYA MEMERLUKAN UPAYA BERSAMA PENUH TEKAD DAN KETULUSAN.

- PENERAPAN DEMOKRASI YANG BERETIKA DAN BERBUDAYA PANCASILA DALAM PILKADA SERENTAK 2018 SANGAT TERGANTUNG PADA KOMPONEN PENYELENGARA, PARTAI POLITIK, PARA KANDIDAT DAN RAKYAT PEMILIH.

- TIGA FAKTOR LAIN YANG BERPENGARUH DALAM MENENTUKAN KADAR DEMOKRASI PILKADA SERENTAK 2018 IALAH REGULASI, SISTEM DAN DUNIA USAHA. 